\title{
HIGH VELOCITY CLOUDS NEAR THE MAGELLANIC CLOUDS
}

\author{
ULRICH MEBOLD \\ Radioastronomisches Institut der Universität Bonn \\ Auf dem Hügel 71 \\ D-5300 Bonn 1 Federal Republic of Germany
}

\begin{abstract}
High velocity clouds (HVCs) of neutral atomic hydrogen close to the position of the Magellanic Clouds (MCs) and the Magellanic Stream (Stream) are reviewed. The gas observed at velocities of $+70 \mathrm{~km} / \mathrm{s}$ and $+130 \mathrm{~km} / \mathrm{s}$ in front of the LMC is probably associated with ordinary HVCs in the galactic halo. This is not the case for the gas observed between +150 and $+170 \mathrm{~km} / \mathrm{s}$ which is more likely associated with the MCs. The HVCs observed superimposed onto the Stream are possibly remnants of collisions between a gaseous polar ring around our Galaxy and the bridge region between the MCs. The HVCs found close to and "behind" the tip of the Stream may be regarded as shreds of the Stream precipitating toward the galactic disk. The chemical composition, the radiation field and the temperature in the Stream is discussed in the context of the first spin temperature determination by Wakker (1990), of an HVC .
\end{abstract}

\section{Introduction}

Since the reviews of the brightness and velocity distribution of HVCs, by Giovanelli (1986) and van Woerden et al. (1986 \& 1985) no major new set of data has become available. The reader is referred to these reviews for comprehensive information.

HVCs are clouds of neutral atomic hydrogen which deviate from co-rotating with the Galaxy by more than $100 \mathrm{~km} / \mathrm{s}$. This definition does not include galaxies like the MCs, but it does include the Stream, although its LSR velocity is close to zero in the region of the south galactic pole. Also, gas falling at a speed of, say $200 \mathrm{~km} / \mathrm{s}$, from a position close to the MC into our Galaxy is included in the HVC concept although its LSR velocity may be rather small. So gas observed towards the MCs in the LSR velocity range between, say +20 and $+300 \mathrm{~km} / \mathrm{s}$, may be HVC gas. As the MCs and the Stream are observed close to a plane normal to the radius vector from the galactic center to the Sun, it makes perfect sense to refer them to the GSR velocities: VGSR $=$ VLSR +250 sinl cosb. HVCs "near" the MCs are here considered as being- a) near in position to the MCs and the Stream, i.e. on the line of sight towards the MCs and the Stream, andb) near in the sense that material from the tip of the Stream may be found to be colliding with the Galaxy at a position more than $180^{\circ}$ away from the MCs.

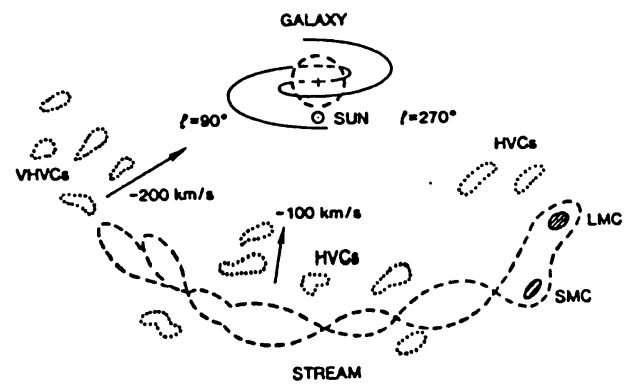

463
Fig. 1. Section of the Stream and the HVCs near the Magellanic System.

R. Haynes and D. Milne (eds.), The Magellanic Clouds, 463-468.

(c) 1991 IAU. Printed in the Netherlands. 
Following a path along the line of sight to the MCs, then along the MS, and finally back down to our Galaxy, we will review the HVCs which are probably located in front of the MCs (sect.2), are superimposed or possibly interacting with the Stream (sect.3), and which are located at the tip of the Stream and possibly raining down on to our Galaxy (sect.4). Finally we will discuss what is known about the chemistry and physical conditions in the Stream compared to HVCs (sect.5).

\section{The HVCs in front of the MCs}

Lines of sight to, or close to, the MCs, sample gas at all velocities from zero to those of the LMC both in emission and in absorption against stars in the MCs. There is a clustering of gas at LSR velocities around $+70 \mathrm{~km} / \mathrm{s}+130 \mathrm{~km} / \mathrm{s}$, and +150 to $+170 \mathrm{~km} / \mathrm{s}$ (Wayte $1990 \mathrm{a}$, de Boer et al. 1990). It is not clear, a priori, at what distances these clouds are located. The gas at $+70 \mathrm{~km} / \mathrm{s}$ because of its large spatial extent is associated with the galactic halo (Wayte 1990a). That at +130 and $+170 \mathrm{~km} / \mathrm{s}$ has been assigned to both the halo and the MCs by various authors. In two recent papers, Wayte (1990a) and de Boer et al. (1990) argue independently that the absorption at +130 $\mathrm{km} / \mathrm{s}$ can be identified with an $\mathrm{HI} 21-\mathrm{cm}$ emission component extending far outside the positional limits of the LMC. From this they conclude that the gas at $+130 \mathrm{~km} / \mathrm{s}$ is not an LMC feature but is an HVC in the galactic halo. Unfortunately the absorption spectra towards the two globular clusters NGC362 (height above the plane $-7 \mathrm{kpc}$ ) and 47 TUC (height above the plane $-3.5 \mathrm{kpc}$ ) by Songaila (1981) do not set upper limits to the HVCs in their directions.

A different result is found by Wayte (1990a) for the gas between +150 and $+170 \mathrm{~km} / \mathrm{s}$. A lack of Ca II absorption at these velocities in the LMC stars Sk-69 52 and Sk-69 103 indicates that this gas is not in front of these stars. This is supported by absorption components towards other LMC stars which are found in the same velocity window and which show an association with the velocity field of the LMC. This led Wayte (1990a) to conclude that the gas between +150 and +170 $\mathrm{km} / \mathrm{s}$ is associated with the LMC.

Finally, this section mentions the narrow, rather elongated dust filaments which seem to be wrap-ping around the LMC (de Vaucouleurs 1955, de Vaucouleurs \& Freeman 1972). McGee $e t$ al. (1986), from the properties of the HI gas, conclude that it is material which is not spatially associated with the LMC. The more prominent one has a proper motion of about $30 \mathrm{~km} / \mathrm{s}$ nearly at right angles to the line of sight to the LMC along a galactic position angle of 2100 . So this filament may be moving out of the plane of the Galaxy at a speed of about $25 \mathrm{~km} / \mathrm{s}$ and may be located anywhere along the line of sight to the MCs - but probably not further out than a few kpc.

\section{The HVCs Superimposed onto the Magellanic Stream}

According to our definition, the Stream as a whole may be regarded as a complex of HVCs. As the Stream itself is adequately covered by other speakers in this conference (Wayte 1990b), I will restrict myself to a discussion of the HVCs superimposed on to the Stream.

The region of the Stream starting at MSI and ranging to MSVI is littered with clouds having both positive and negative anomalous velocities compared to the Stream (Haynes 1979, Cohen 1982, Morras 1983, 1985). The sizes of these clouds range from about $10^{\circ}$ down to the angular resolution employed for the observations. The velocities found differ by more than $200 \mathrm{~km} / \mathrm{s}$ from that of the Stream. Cohen and also Morras note strong velocity gradients in some of the clouds.

Various interpretations have been proposed for these HVCs. They range from intergalactic gas as-sociated with the Sculptor group galaxies NGC55 and NGC300 (Mathewson et al. 1975, 
Haynes 1979) to HVCs which are either unrelated to, or interacting with, the Stream. Mathewson et al. (1987) and Wayte (1990b) stress the significance of the velocity gradients observed in the Stream and the associated HVCs as evidence for an origin of the Stream by ram pressure sweeping. Inelastic collisions of HVCs orbiting in the galactic halo with the gaseous bridge between the MCs will cause the gas in the bridge to lose part or all of its transverse orbital momentum. It will start falling towards the Galaxy. The tip of the Stream is falling fastest because it is the first part to have been swept out immediately after a hypothesized collision between the MCs about $4 * 10^{8}$ years ago (Wayte $1990 \mathrm{~b}$ ). The observed velocity gradients in this model are a natural consequence of systematic density gradients in the colliding partners. The large velocity scatter of some HVCs around the Stream are consistently explained by the scatter of the collision parameters for the inelastic collisions of the HVCs and clumps in the density distribution of the Magellanic bridge.

In fact, ram pressure sweeping of the Magellanic bridge by a quasi-homogeneous halo gas with embedded HVCs can explain many of the observed features of the Stream and its HVC environment. But there are problems with the space density of the HVCs close to the orbit of the MCs if the HVCs are randomly distributed in the galactic halo and the local group of galaxies, as in the models of Wakker (1990). In this case the probability of collisions between the Magellanic bridge and HVCs will be too small. Also, the fairly regular spacings between the subsystems MSI to MSVI of the Stream pose a problem if the HVCs are randomly distributed. The space density problem may be overcome if the HVCs are concentrated in a kind of polar ring around our Galaxy at about the distance of the MCs, as proposed by Haud (1988). The regular spacings of MSI to MSVI may be indicative of turbulent eddies in the wake of the MCs. However, detailed hydrodynamical modelling is required to verify this kind of speculation.

\section{The HVCs at the Tip of the Magellanic Stream}

North of galactic latitude -650 , the Stream shows an even more complex structure than in its parts closer to the MCs. It again contains filaments on all scales from about $10^{\circ} \mathrm{down}$, and widens to about twice the width of its more southerly sections. North of latitude $-60^{\circ}$ the surface brightness of the HI emission drops considerably and the Stream takes on a strongly fragmented appearance. Even further to the north the very high velocity clouds (VHVCs) defined by their extreme velocities with respect to the LSR (VLSR $=-300$ to $-450 \mathrm{~km} / \mathrm{s}$ ), are found scattered over the whole area of the first and second galactic quadrant; the GSR velocities drop to $-200 \mathrm{~km} / \mathrm{s}$. The VHVCs found in this area of the sky may be regarded as shreds of the Stream precipitating toward the galactic disk (e.g. Giovanelli 1981, Cohen 1982). The widening of the Stream close to its tip is possibly a projection effect caused by its relatively small distance from the observer. The disintegration of the Stream close to its tip and behind is possibly an effect of the hydrodynamical friction on the surface of the clouds which are falling at high speeds through the galactic halo gas. A verification of the concept of shreds falling from the tip of the Stream into the galactic disk requires finding evidence for an interaction with the gas in the galactic halo or even with the galactic disk. We do not have any evidence for an interaction of these HVCs with the halo gas but we expect that ROSAT will show the hot gas in the bow-shock regions if the model is valid.

There is, however, some evidence for collisions of HVCs with the galactic disk. In Table 1 names, positions and references for proposed collision sites are collected. The evidence for these collisions is of varying quality but it appears likely that at least some of them really are collisions. The nature of this evidence is similar for all entries in Table 1 except for M1 and the last entry. It consists essentially of close positional coincidences and anti-coincidences of low, intermediate and 
high velocity gas and bridges of $\mathrm{HI}$ emission between these velocity components. For more details see the review of Mebold $e t$ al. (1990). M1 is considered a candidate for an interaction with the gas in the halo because of its head-tail structure in the HI brightness and velocity distributions (Giovanelli et al. 1973). The inclusion of the Orion nebula and the Monoceros complex in this list is debatable. It refers to a suggestion of Franco et al.(1988) to explain why isolated, massive molecular cloud complexes like the ones listed are located rather far outside the galactic plane.

Table 1. Suspected HVC/Galaxy Collision Sites

\begin{tabular}{|c|c|c|}
\hline Name/s & $\begin{array}{l}\text { Position } \\
\left(1^{0}, \quad \text { b }\right)\end{array}$ & Reference \\
\hline IVC86+40 & $86,+40$ & Mebold et al. 1989 \\
\hline Draco nebula & $91,+38$ & Rohlfs et al. 1989 \\
\hline $\begin{array}{l}\text { Cohen filament/ } \\
\text { part of GS120-30 }\end{array}$ & $120,-30$ & $\begin{array}{l}\text { Cohen } 1981 \\
\text { Heiles } 1984\end{array}$ \\
\hline $\begin{array}{l}\text { Wesselius shell/ } \\
\text { part of GS155+38 }\end{array}$ & $155,+38$ & $\begin{array}{l}\text { Wesselius } 1973 \\
\text { Heiles } 1984\end{array}$ \\
\hline M1 & $165,+66$ & Giovanelli et al. 1973 \\
\hline $\begin{array}{l}\text { Anticenter shell } \\
\text { or GS174+02 }\end{array}$ & $174,+02$ & $\begin{array}{l}\text { Heiles } 1984 \\
\text { Mirabel } 1982\end{array}$ \\
\hline $\begin{array}{l}\text { Orion/Monoceros } \\
\text { complexes }\end{array}$ & $200,-30$ & Franco et al. 1988 \\
\hline
\end{tabular}

It is hardly possible to prove that any of these colliding HVCs have come from the Magellanic System. But the possible collision sites known so far are all located in the first and second galactic quadrant. It has to be checked whether this is an observational selection effect.

\section{Chemical Composition and Physical Conditions in the Stream}

If the Stream has been swept out of the bridge region of the MCs by collisions with HVCs or other gas in the galactic halo, the gas is a mixture of LMC, SMC and HVC gas and we would expect the heavy element abundances to reflect that mixture. The dust is probably destroyed by sputtering during the collision process. The temperature of the gas in the Stream depends on the strength of the ambient radiation field and on the abundance of the heavy elements which are the essential ingredients for the heating and cooling processes to work in the interstellar medium.

Following the original study of Songaila (1981), Penston (1982) confirmed the original detection of $\mathrm{Ca}$ II towards MSI and also found interstellar lines of HI, CII, CIV, OI, MgII, SiII, and FeII in the line of sight towards the galaxy Fairall 9. The deduced abundances cannot, however, be used to distinguish between a primordial, an MC or a galactic origin of the gas in the Stream (West $e t$ al. 1985). There are only marginal amounts of dust in the Stream, if any. Fong $e t$ al. (1987) used galaxy counts and the IRAS data base and found no significant evidence for extinction and no evidence at all for IR emission associated with the Stream. But they investigated only a small part of MSII so that this result can only be regarded as preliminary.

If there is dust in the Stream, the radiation field must be very diluted as no IR emission is observed. Then the spin temperature of the HI gas in the Stream is expected to be very low. If, on the other hand, there is no dust in the Stream, the most effective heating process in our Galaxy, the 
heating by the photo effect on dust grains, does not work even if there is an appreciable radiation field. Again, the spin temperature is expected to be low. So, probing HI spin temperatures in the MCs and the Stream by HI absorption observations against extra galactic background radio sources might give valuable information about the radiation field and the dust content.

Attempts to derive spin temperatures in HVCs with northern telescopes have been unsuccessful until very recently because even the largest single-dish telescopes are too limited in angular resolution. Payne et al. (1980) succeeded in deriving spin temperatures of about $100 \mathrm{~K}$ or less for IVC gas $(-100<\mathrm{Vlsr}<50 \mathrm{~km} / \mathrm{s})$ on only a few sight lines towards extragalactic radio sources by using the Arecibo radio telescope. A recent survey of $\mathrm{HI}$ absorption in IVCs and HVCs with the same telescope (Colgan et al. 1990) resulted in lower spin-temperature limits of only $70 \mathrm{~K}$ or less for HVCs and of $1000 \mathrm{~K}$ or less for IVCs. Wakker (1990), by using the Westerbork Synthesis Radio Telescope, has found $\mathrm{HI}$ absorption in an HVC for the first time. He found temperatures of about $40 \mathrm{~K}$ for two clumps in HVC 131+1-200.

Thus, the range of temperatures found for HVCs is quite similar to that established for HI clouds in the galactic disk. Taking into account that HVCs were found to have a core-halo morphology (cf. e.g. Giovanelli and Haynes 1976, Cohen and Mirabel 1979) similar to that of disk HI clouds, we are facing an unexpected result. The heating-cooling balance for HVCs results in about the same temperature and density structures as that for clouds in the galactic disk, although this balance is probably strongly offset in the two species of clouds. A reduced heat input into the HVC gas, caused by a strongly diluted radiation field, is obviously compensated by strongly reduced cooling, possibly caused by a lack of cooling agents, like heavy ions. This problem is discussed more extensively by Colgan et al. (1990).

Finally, this leads us to ask: are the Stream and HVCs indeed closely related phenomena? In the Stream the core-halo morphology which was found to be typical for HVCs is not observed (Mathewson et al. 1974, Mirabel et al. 1979, McGee et al. 1983). This observational difference is not understood and may point to basic differences either in the corresponding distances or in the physical conditions of the gas, such as temperature, density chemical composition or the intensity of the ambient radiation field (West et al. 1985).

As there are probably no stars either in the Stream or in the HVCs, the ambient radiation field in the Stream and HVCs is probably not much different but is dominated by the intergalactic radiation field. So, if there is a difference, it is probably related to the metal abundance. For instance, the HI clouds in the Stream may not have cold cores because of a lack of cooling agents.

An answer could probably be obtained by experiment. The Australia Telescope can be used to determine the temperature of the gas, so that further information on the dust content and the radiation field in the Stream and in related or unrelated southern HVCs can be obtained.

\section{Acknowledgments}

I would like to thank R.F. Haynes and his family for hospitality during the IAU conference and the Australia Telescope National Facility for support during my sabbatical visit to Epping.

\section{References}

de Boer, K.S., Morras, R. \& Bajaja, E.(1990), Astron. Astrophys. 233, 523.

Cohen, R.J. (1981), Mon. Not. R. Astron. Soc. 196, 835.

Cohen, R.J. (1982), Mon. Not. R. Astron. Soc. 200, 391.

Cohen, R.J. \& Mirabel, I.F. (1979), Mon. Not. R. Astron. Soc. 186, 217.

Colgan, S.W.J., Salpeter, E.E. \& Terzian, Y. (1990), Astrophys. J. 351, 503. 
Fong, R., Jones, L.R., Shanks, T., Stevenson, P.R.F., Strong, A.W., Dawe J.A. \& Murray J.D. (1987), Mon. Not. Astron. Soc. 224, 1059.

Franco, J., Tenorio-Tagle, G., Bodenheimer, P., Rozyczka, M. \& Mirabel, I.F. (1988), Astrophys.J. 333, 826.

Giovanelli, R., Verschuur, G.L. \& Cram, T.R. (1973, Astron. Astrophys. Suppl. 12, 209.

Giovanelli, R. (1981), Astron. J., 86,1468.

Giovanelli, R. (1986), Gaseous Halos of Galaxies, NRAO Workshop No. 12, Green Bank, W.Va., J.N. Bregman \& F.J.Lockman (eds) p.99.

Giovanelli, R. \& Haynes, M.P. (1976), Mon. Not. R. Astron. Soc. 177, 525

Haud, U.(1988), Astron. Astrophys.. 198, 125.

Haynes, M.P. (1979), Astron. J. 84, 1173.

Heiles, C. (1984), Astrophys. J. Suppl. 55, 585.

Mathewson, D.S., Cleary,.M.N. \& Murray, J.D.(1974), Astrophys.J. 190, 291.

Mathewson, D.S., Cleary,.M.N., Murray, J.D. (1975), Astrophys.J. 195, L97.

Mathewson, D.S., Wayte, S.R., Ford, V.L. \& Ruan, K. (1987), Proc. Astron. Soc. Austr. 7, 19.

McGee, R.X., Newton, L.M. \& Morton, D.C. (1983), Mon. Not. R. Astron. Soc. 205, 1191.

Mebold, U., de Boer, K.S., Wennmacker, A. (1990), Proc. 11 th Europ. Reg. Astron. Meeting, Tenerifa, in press.

Mebold, U., Herbstmeier, U., Kalberla, P.M.W. \& Souvatzis, I. (1989), Structure and Dynamics of the Interstellar Medium, G.Tenorio-Tagle et al.(eds), IAU Coll. 120 in press.

Mirabel, I.F. (1982), Astrophys. J. 256, 112.

Mirabel, I.F., Cohen, R.J. \& Davies, R.D. (1979), Mon. Not. R. Astron. Soc. 186, 433.

Morras, R. (1983), Astron. J. 88, 62.B

Morras, R. (1985), Astron. J. 90, 1801.

Payne, H.E., Salpeter, E.E. \& Terzian, Y. (1980), Astrophys. J. 240, 499.

Penston, M.V. (1982, The Observatory 102, 174.

Rohlfs, R., Herbstmeier, U., Mebold, U. \& Winnberg, A.(1989), Astron. Astrophys. 211, 402.

Songaila, A. (1981), Astrophys. J. 243, L19.

de Vaucouleurs, G. (1955), Astron. J. 60, 126.

de Vaucouleurs, G. \& Freeman K.C.(1972), Vistas Astron. 14, 163.

Wakker, B. (1990), Ph.D. Thesis, Univ. Groningen.

Wayte, S.R. (1990a), Astrophys. J. 355, 473.

Wayte, S.R. (1990b), this volume.

Wesselius, P.R. \& Fejes, I. (1973), Astron. Astrophys. 24, 15.

West, K.A., Pettini, M., Penston, M.V., Blades, J.C. \& Morton, D.C. (1985), Mon. Not. R. Astron. Soc. 215, 481.

Van Woerden , H., Schwarz, U.J. \& Hulsbosch, A.N.M. (1985) IAU Symposium 106, The Milky Way Galaxy, van Woerden et al. (eds) (Reidel: Dordrecht) p.387.

Van Woerden,H., Schwarz, U.J., Robertson, J.G. \& Hulsbosch, A.N.M. (1986), Gaseous Halos of Galaxies, NRAO Workshop No. 12, Green Bank W.Va, J.N. Bregman \& F.J. Lockman, (eds) p.115. 